\title{
ANÁLISIS DE LAS MODIFICACIONES EN LA HIDRODINÁMICA EN UN TRAMO DEL RIO PARANÁ INFERIOR MEDIANTE LA APLICACIÓN DEL MODELO MATEMÁTICO TELEMAC-2D
}

\author{
Florencia Peruzzo $^{(1)(2)(4)}$ Gerardo Riccardi $^{(1)(2)(3)}$, Pedro A. Basile ${ }^{(1)(2)}$ \\ ${ }^{(1)}$ Departamento de Hidráulica - Escuela de Ingeniería Civil (FCEIA - UNR) \\ ${ }^{(2)}$ Centro Universitario Rosario de Investigaciones Hidroambientales (FCEIA - UNR) \\ ${ }^{(3)}$ Consejo de Investigaciones de la Universidad Nacional de Rosario \\ ${ }^{(4)}$ Consejo Nacional de Investigaciones Científicas y Técnicas \\ Riobamba 245 bis. (2000) Rosario. Argentina \\ e_mail: fperuzzo@fceia.unr.edu.ar
}

\begin{abstract}
RESUMEN
En este trabajo se presenta la implementación de un modelo de simulación bidimensional empleando una grilla triangular irregular para caracterizar la hidrodinámica de un tramo del río Paraná Inferior comprendido entre el km 410 y el km 452 de la ruta de navegación Santa Fe - Océano. Se crearon dos constituciones a partir de relevamientos batimétricos realizados en los períodos de años 2004-2006 y 2010-2012 y se realizaron simulaciones para estados de flujo permanente e impermanente con el modelo bidimensional Telemac-2D. En flujo permanente se utilizaron caudales aforados comprendidos entre $11640 \mathrm{~m}^{3} / \mathrm{s}$ y 28600 $\mathrm{m}^{3} / \mathrm{s}$, mientras que, en flujo impermanente se empleó la serie de caudales registrada en el período 01/01/2004 al 31/12/2004 y en el período 16/03/2015 al 15/03/2016 para cada constitución respectivamente. Las variables de ajuste fueron los niveles observados en los hidrómetros de Rosario (ROS-km 416) y Puerto San Martín (PSM-km 448), empleándose como parámetro de calibración el coeficiente de rugosidad de Manning. Además se contrastaron caudales y velocidades con los aforados por la Facultad de Ingeniería y Ciencias Hídricas de la UNL en 2004-2006 y por la Universidad de Bologna en 2009, obteniendo una satisfactoria aproximación. Luego se compararon las particiones de caudales en las cuatro bifurcaciones del tramo, observándose tanto en las mediciones como en las simulaciones un incremento de los porcentajes del caudal total derivado por el brazo derecho.
\end{abstract}

Palabras clave: Modelación bidimensional; ríos aluviales de llanura; Río Paraná; Telemac-2D

\begin{abstract}
In this paper the implementation of a two-dimensional simulation model using a triangular irregular mesh to characterize the hydrodynamics of a reach of the Low Parana River between the $\mathrm{km} 410$ and the $\mathrm{km} 452$ of the Santa Fe - Ocean waterway is presented. Two constitutions were created from bathymetric surveys carried out in the periods of years 2004-2006 and 2010-2012 and steady and unsteady flow simulations were performed with the two-dimensional model Telemac-2D. In steady flow measured discharges between 11640 $\mathrm{m}^{3} / \mathrm{s}$ and $28600 \mathrm{~m}^{3} / \mathrm{s}$ were used, whereas in unsteady flow the series of discharges registered in the period $01 / 01 / 2004$ to $31 / 12 / 2004$ and in the period $16 / 03 / 2015$ to $15 / 03 / 2016$ were considered for each model constitution respectively. The variables of adjustment were the water levels observed at Rosario (ROS-km 416) and Puerto San Martin (PSM-km 448), using the Manning roughness coefficient as a calibration parameter. Moreover, discharges and velocities were compared with those measured by the Faculty of Engineering and Water Sciences (FICH) and by the University of Bologna, obtaining a good approximation. Then the flow partitions were compared in each constitution in the four bifurcations of the reach, observing an increase of the percentages of the total flow derived by the right arm.
\end{abstract}

Keywords: Two-dimensional modelling; lowland alluvial rivers; Paraná River; Telemac-2D. 


\section{INTRODUCCIÓN}

Los grandes ríos aluviales de llanura representan un recurso natural de inestimable valor ambiental para un territorio, por ello la sustentabilidad de tales ambientes fluviales resulta de vital importancia. El río Paraná, río aluvial que recorre la zona litoral de nuestro país, forma parte de la Cuenca del Plata, junto con los ríos Paraguay y Uruguay, sus afluentes y varios humedales como los Esteros del Iberá, el Bañado la Estrella y la gran planicie aluvial propia del río. Las aguas superficiales de dicha cuenca son recolectadas principalmente por el denominado Corredor Fluvial Paraná - Paraguay, que se destaca por la presencia de grandes extensiones de humedales (SAyDS, 2013). Respecto de la superficie total de la Cuenca del Plata, $3.170 .00 \mathrm{~km}^{2}$, el Paraná drena una superficie de $2.600 .00 \mathrm{~km}^{2}$ a lo largo de sus 4.000 kilómetros de longitud (Taller ecologista, 2010). La magnitud de los volúmenes de escurrimiento y la longitud de su recorrido hacen que el mismo se encuentre entre los ríos de llanura más importantes del mundo.

El río Paraná tiene usos múltiples entre los que se destacan el aprovechamiento hidroeléctrico, el abastecimiento de agua, el uso como vía de comunicación para navegación comercial y recreacional, entre otros. En el Bajo Paraná, formado por el Paraná Medio y el Paraná Inferior, se desarrolla la mayor parte de las actividades agrícolas e industriales del país, cerca del $70 \%$ de su población se asienta en esta zona (SAyDS, 2013). En este contexto de interacción entre las actividades humanas y el recurso fluvial es necesario por lo tanto contar con herramientas computacionales adecuadas para describir los procesos hidrodinámicos en el cauce principal a los efectos de contar con una robusta base hidrodinámica para describir otros procesos asociados como el tránsito de sedimentos, la dispersión de contaminantes, procesos asociados con la biota del sistema fluvial y la morfodinámica del sistema. Para la descripción de la hidrodinámica, una herramienta ampliamente utilizada en grandes dominios espacio-temporales de cálculo es la modelación matemática a superficie libre, que ha evolucionado paralelamente a la capacidad de los ordenadores, al desarrollo del cálculo numérico y a la posibilidad de obtener mayor cantidad de datos. Actualmente, la modelación hidrodinámica se ha convertido en una herramienta esencial para la planificación y gestión de los recursos hídricos como también para cuantificar el impacto de las actividades humanas para la protección de los mismos y del medio ambiente (Singh y Woolhiser, 2002).

El tramo del río Paraná que se estudia en este trabajo abarca desde el $\mathrm{km} 452$ hasta el $\mathrm{km} 410$ de la ruta de navegación troncal Santa $\mathrm{Fe}$ - Océano. Existen antecedentes de simulaciones con modelos matemáticos aplicados satisfactoriamente en el tramo, tales como, modelos bidimensionales completos (FICH, 2006; Guerrero y Lamberti, 2013; Basile et al., 2015) y modelos cuasi-bidimensionales de baja complejidad (Peruzzo et al., 2016). Asimismo Garcia et al. (2013) ha llevado adelante una modelación cuasi $2 \mathrm{D}$ en cauce principal y planicie de inundación en un tramo de $208 \mathrm{~km}$ entre la ciudades de Diamante y Ramallo, incluyendo el tramo en estudio.

\section{OBJETIVOS}

El objetivo de este trabajo es evaluar, mediante la simulación numérica bidimensional, las modificaciones en la hidrodinámica a partir de la evolución morfológica en un tramo del río Paraná Inferior entre el $\mathrm{km} 410$ y el km 452, en los periodos 2004-2006 y 2010-2012. Para esto se procedió a calibrar y validar dos constituciones establecidas a partir de relevamientos batimétricos realizados en los períodos mencionados anteriormente y se analizó en detalle la capacidad del modelo de reproducir la partición de caudales en las cuatro bifurcaciones del tramo.

\section{TRAMO EN ESTUDIO}

El área en estudio, la cual se presenta en la Figura 1, se limitó a un tramo del río Paraná Inferior comprendido entre el $\mathrm{km} 410$ y el km 452 de la ruta de navegación Santa $\mathrm{Fe}$ - Océano contando con aproximadamente $42 \mathrm{~km}$ de extensión. El cauce principal en este tramo se desarrolla sobre la margen santafesina abarcando distintas localidades como Puerto San Martin, San Lorenzo, Fray Luis Beltrán, Capitán Bermúdez, Granadero Baigorria, Rosario y Villa Gobernador Gálvez, a lo largo de la cual se desarrolla un importante cordón industrial junto con la presencia de numerosas instalaciones portuarias. El tramo comprende la mayor parte de la infraestructura portuaria del Área Metropolitana Gran Rosario, que se desarrolla desde la localidad de Timbúes hasta Arroyo Seco, por donde se despachan entre el $78 \%$ y el $80 \%$ de las exportaciones 
argentinas de aceites, granos y subproductos. La mayor parte de estas exportaciones son productos del complejo sojero (BCR, 2015).

En este tramo, el río fluye en una dirección predominante norte-sur presentando una configuración cauce principal e islas, caracterizándose con un ancho medio para el tramo completo de $1580 \mathrm{~m}$ (FICH, 2004). El valle de inundación se desarrolla sobre la margen izquierda del río y el ancho del mismo en esta zona varía entre $40-50 \mathrm{~km}$. Esta gran planicie asociada se inunda parcial o totalmente durante las crecidas. El caudal medio anual es de $17500 \mathrm{~m}^{3} / \mathrm{s}$ según caudales registrados en Puerto San Martin (PSM, $\mathrm{km} \mathrm{448)} \mathrm{durante} \mathrm{el} \mathrm{período} \mathrm{1970-2014} \mathrm{(Basile} \mathrm{et}$ al., 2015). Durante la crecida extraordinaria de 1983 el mayor caudal de agua fue cerca de 60000 $\mathrm{m}^{3} / \mathrm{s}$, casi $30000 \mathrm{~m}^{3} / \mathrm{s}$ circulaban sobre el cauce principal (Garcia et al., 2013). Para dicho evento, el nivel máximo de agua en Rosario (ROS, km 416) fue de $9.21 \mathrm{~m}$ IGN y el valle estuvo completamente inundado con una profundidad media de $4 \mathrm{~m}$ de agua. Mientras que en PSM el menor caudal registrado fue alrededor de $7000 \mathrm{~m}^{3} / \mathrm{s}$ (Basile et al., 2015). La pendiente hidráulica que se verifica en el tramo es de $1.4 \times 10^{-5} \mathrm{~m} / \mathrm{m}$ para aguas bajas y de $4.3 \times 10^{-5} \mathrm{~m} / \mathrm{m}$ para aguas altas.

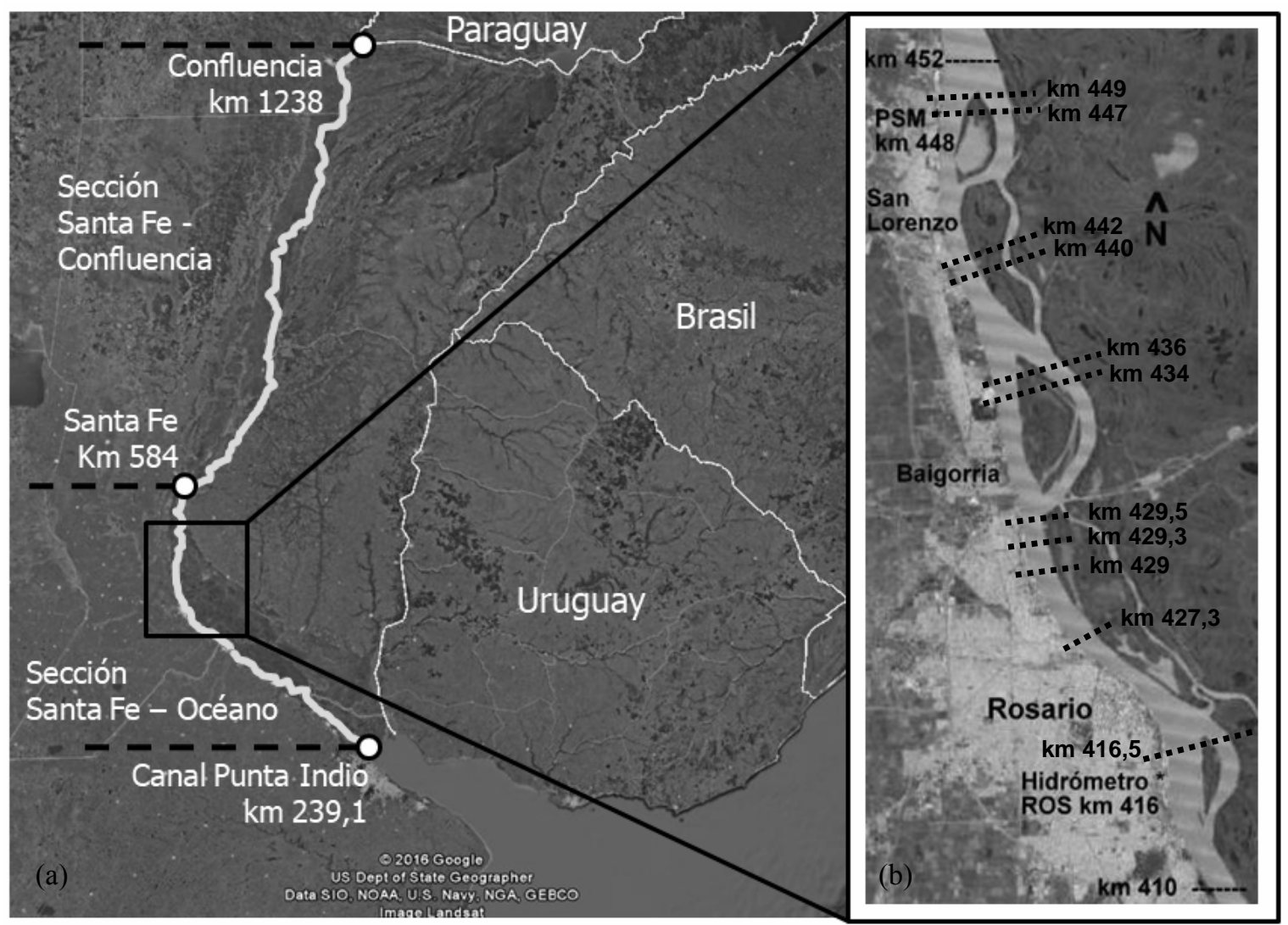

Figura 1. (a) Vía Navegable Troncal Confluencia - Océano; (b) Ubicación del tramo en estudio comprendido entre el km 410 y el km 452 de la ruta de navegación Santa Fe - Océano. Google Earth@.

\section{MATERIALES Y METODOS}

\section{Modelo matemático TELEMAC-2D}

El modelo de simulación implementado es el TELEMAC-2D (Hervouet, 2000) acoplado a la plataforma de pre y postprocesamiento de datos y resultados bajo entorno de ventanas Blue KenueC (CHC-NRC, 2011). TELEMAC-2D es un modelo bidimensional de uso gratuito, basado en las ecuaciones para aguas poco profundas bidimensionales en horizontal completas, las cuales son resueltas numéricamente mediante el método de elementos finitos (Galland et al., 1991). Las ecuaciones son (Lang et al., 2014):

Continuidad:

$$
\frac{\partial h}{\partial t}+u \cdot \vec{\nabla}(h)+h \operatorname{div}(\vec{u})=S_{h}
$$


Momentum en dirección $\mathrm{x}$ :

$$
\frac{\partial u}{\partial t}+\vec{u} \cdot \vec{\nabla}(u)=-g \frac{\partial Z}{\partial x}+S_{x}+\frac{1}{h} \operatorname{div}\left(h v_{t} \vec{\nabla} u\right)
$$

Momentum en dirección y:

$$
\frac{\partial v}{\partial t}+\vec{u} \cdot \vec{\nabla}(v)=-g \frac{\partial Z}{\partial y}+S_{y}+\frac{1}{h} \operatorname{div}\left(h v_{t} \vec{\nabla} v\right)
$$

Donde: $\mathrm{h}$ es la profundidad de flujo; $\mathrm{g}$ es la aceleración de la gravedad; $v_{t}$ es el coeficiente de difusión; $Z$ es el nivel de la superficie libre; $t$ es el tiempo; $S_{h}$ es una fuente o sumidero en la ecuación de continuidad; $\vec{\nabla}$ es el gradiente; div es la divergencia; $\mathrm{u} y \mathrm{v}, \mathrm{S}_{\mathrm{x}}$ y $\mathrm{S}_{\mathrm{y}}$ son las velocidades medias en vertical y las fuentes o sumideros en las ecuaciones de cantidad de movimiento en el sentido $\mathrm{x}$ e y respectivamente.

\section{Implementación del modelo matemático TELEMAC-2D}

La aplicación del modelo se inició con la definición topobatimétrica del cauce principal. Para esto se constituyó el modelo digital del terreno del fondo del río, las márgenes e islas de dos escenarios morfológicos correspondientes a los períodos de años 2004-2006 y 2010-2012 a partir de datos existentes recopilados de relevamientos realizados por la Facultad de Ingeniería y Ciencias Hídricas - UNL (FICH, 2004 y 2006) y por la Dirección Nacional de Vías Navegables Delegación Paraná Inferior (DNVN-DPI, 2014) respectivamente. Se digitalizaron las curvas de nivel del fondo georeferenciadas al sistema de coordenadas POSGAR 94 Faja 5 Datum WGS 1984 con cotas referidas al cero IGN. Se ajustó la base espacial del área de modelación mediante un mosaico construido a partir de 4 cartas náuticas provistas por el Servicio de Hidrografía Naval (SHN, 2014) [Figura 2(a)]. Luego, se crearon dos representaciones espaciales del fondo del cauce principal utilizando mallas computacionales. Mediante el software Blue Kenue $\mathrm{C}$, se construyeron dos grillas triangulares irregulares con una distancia máxima entre nodos igual a $50 \mathrm{~m}$, permitiendo capturar toda la información disponible de los relevamientos existentes. La constitución M04-06, obtenida a partir de los relevamientos de la $\mathrm{FICH}$, quedó conformada por 40712 nodos y 79501 elementos. Mientras que la constitución M10-12, elaborada sobre la base de la información relevada por DNVN-DPI, quedó conformada por 67093 nodos y 132124 elementos. En la Figura 1(b) se presenta una imagen de ambas constituciones donde se observan los niveles del lecho expresados en $\mathrm{m}$ referidos al cero IGN. Vale aclarar que la constitución M10-12 se extendió hasta el km 405 para incluir la vinculación con el riacho La Brava.

Para cada constitución se realizaron simulaciones en flujo permanente usando un rango de caudales entre $11640 \mathrm{~m}^{3} / \mathrm{s}$ hasta $28600 \mathrm{~m}^{3} / \mathrm{s}$ (aguas bajas a altas). Los caudales empleados en las simulaciones corresponden a caudales aforados por la Facultad de Ingeniería y Ciencias Hídricas (FICH, 2004 y 2006) y por la Subsecretaría de Recursos Hídricos de la Nación en la estación Paraná-Timbúes. Además, se realizaron simulaciones en flujo impermanente reproduciendo las series de caudales observadas en la estación ParanáTimbúes en el período 01/01/2004 al 31/12/2004 para la constitución M04-06 y en el período 16/03/2015 al 15/03/2016 para las constitución M10-12, abarcando esta última la crecida del río Paraná de los meses de enero y febrero de 2016. El parámetro de calibración considerado fue el coeficiente de rugosidad de Manning $\eta$ y las variables de ajuste fueron los niveles de agua observados en los hidrómetros de Puerto San Martin (PSM, km 448) y Rosario (ROS, km 416).

Para las simulaciones en flujo permanente, los caudales aforados fueron ingresados como condición de borde aguas arriba y la condición de borde aguas abajo se constituyó con la correspondiente altura de agua asociada al caudal entrante. Esta altura de agua se obtuvo extrapolando niveles desde el Hidrómetro de Rosario empleando la pendiente de la superficie libre calculada entre PSM y ROS para la fecha de aforo. Para las simulaciones en flujo impermanente, la condición de borde aguas arriba fue la serie de caudales diarios registrados en la estación Paraná-Timbúes en el período 01/01/2004 al 31/12/2004 para la constitución M04-06, y para M10-12 la serie registrada en el período 16/03/2015 al 15/03/2016. En lo que respecta a la condición de borde aguas abajo para ambas constituciones se empleó una relación altura-caudal.

Los resultados de las simulaciones en flujo permanente de la constitución M04-06 se contrastaron con mediciones de caudales en las cuatro bifurcaciones que presenta el tramo en estudio: 1. Isla El Banco-km 449; 2. Isla Rosita-km 440; 3. Isla Carlota-km 436 y 4. Isla Ing. Sabino Corsi-km 416.5. Para cada uno de los 7 escenarios de flujo permanente simulados se estimaron los caudales derivados por el brazo derecho e izquierdo en estas secciones y se compararon con caudales aforados por FICH (2004 y 2006). También se compararon con los caudales estimados en el trabajo de Basile et al. (2015) y Peruzzo et al. (2016). Las cuatro bifurcaciones consideradas se muestran en la Figura 1b. Además, 
los resultados se compararon con distribuciones transversales de caudales específicos observadas en mediciones de campo con ADCP realizadas también por la FICH en el período noviembre-diciembre 2004 y febrero-marzo 2006 (FICH, 2006) en distintas secciones transversales a lo largo del dominio. Por su parte, los resultados de la constitución M10-12 se contrastaron con caudales aforados en las bifurcaciones 1 a 3 y con perfiles transversales de velocidad medidos con $\mathrm{ADCP}$ durante la campaña realizada en conjunto entre la Universidad de Bologna (UNIBO) y la Facultad de Ingeniería y Ciencias Hídricas (FICH) los días 29 de junio a 3 de julio de 2009 (Kazimierski, 2014).

Para el análisis de la evolución de la hidrodinámica se estudió la partición de caudales en las cuatro bifurcaciones del dominio. Se compararon caudales simulados derivados por ambos brazos en cada bifurcación para ambas constituciones.

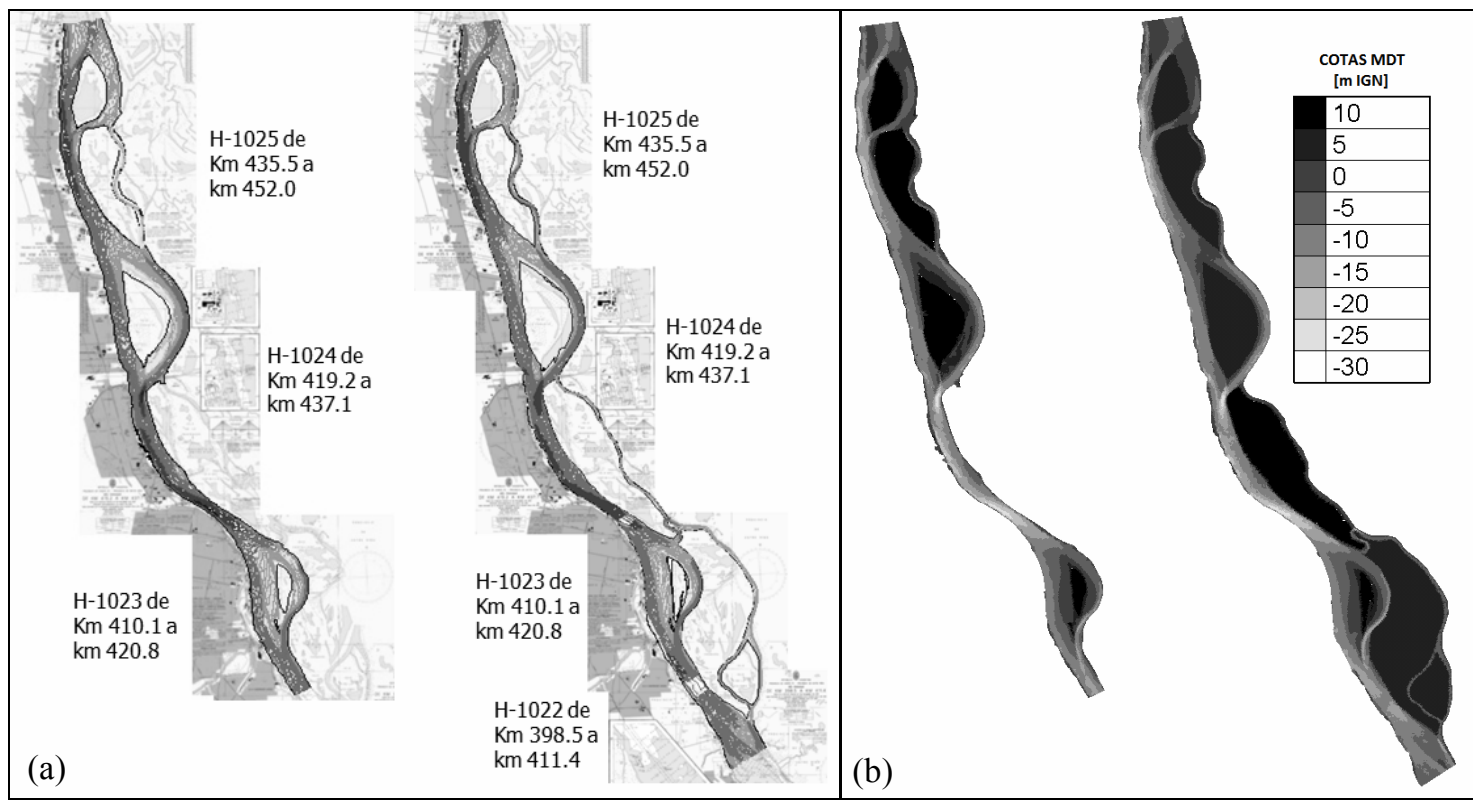

Figura 2. (a) Curvas de nivel digitalizadas según relevamientos de FICH (2004-2006) y DNVN-DPI (2010-2012) junto con las cartas náuticas del SHN; (b) Constitución del modelo M04-06 y M10-12 y MDT.

\section{EVALUACIÓN DE RESULTADOS}

\section{Resultados de las simulaciones de la Constitución M04-06}

En flujo permanente, el rango de valores del coeficiente de rugosidad de Manning que proporcionó el mejor ajuste en términos de niveles estuvo comprendido entre 0.025 para aguas bajas y 0.024 para aguas altas. El error relativo porcentual entre niveles observados y simulados se acota en $2.07 \%$, siendo el valor medio $0.59 \%$. Los niveles calculados se encuentran dentro del entorno de variación obtenido a partir de caudales y niveles aforados por la Subsecretaría de Recursos Hídricos de la Nación en la estación Paraná-Timbúes en el período 2000-2016. En la Tabla 1 se muestra una síntesis de los parámetros observados y calculados para cada simulación.

A los efectos de estudiar la reproducción de la partición del caudal total en las cuatro bifurcaciones que presenta el tramo de río en estudio, se procedió en primera instancia a estimar los caudales derivados en las distintas secciones para cada uno de los 7 escenarios de flujo permanente simulados. Estas secciones, que se indican en la Figura 1, corresponden a: 1. Isla El Banco-km 449, 2. Isla Rosita-km 440, 3. Isla Carlotakm 436 y 4. Isla Ing. Sabino Corsi-km 416.5. En la Tabla 2 se presentan los porcentajes del caudal total Q derivados en cada bifurcación para el brazo derecho (BD) y para el brazo izquierdo (BI), para cada simulación. Se observa que en las secciones correspondientes a los $\mathrm{km} 449,440$ y 436 el porcentaje del caudal total derivado por el brazo derecho es superior para aguas bajas que para aguas altas, mientras que en la sección correspondiente al $\mathrm{km} 416.5$ este porcentaje es similar para ambos estados. En las cuatro secciones analizadas el brazo derecho corresponde al curso principal. Los caudales aforados por FICH (2004 y 2006) en las bifurcaciones corresponden a caudales distintos a los empleados en las simulaciones para la etapa de calibración. Para poder determinar los valores 
a comparar con los aforos, se procedió a ajustar relaciones lineales entre caudal total Q simulado versus los respectivos valores de porcentaje de caudal derivado por cada brazo en cada bifurcación. Una vez halladas estas relaciones se obtuvieron los porcentajes en función de los caudales aforados para luego calcular los errores relativos de su comparación con los porcentajes observados. Dado que se dispone de dos aforos por cada bifurcación, se estableció también un promedio de los valores absolutos de los errores porcentuales (PVAEP) para cada una. Para completar el análisis se contrastaron también los porcentuales de derivación con los que arrojaran los trabajos de modelación de Basile et al. (2015) y Peruzzo et al. (2016). En la Tabla 3 se presentan los resultados de las comparaciones realizadas en el brazo derecho para cada bifurcación. Se observa que el modelo distribuye adecuadamente los caudales en las bifurcaciones que presenta el tramo de río en estudio. Analizando caudales observados y aforados se aprecia que el valor del error relativo máximo se acota en $5.22 \%$. El valor medio de los errores relativos porcentuales entre porcentajes de caudales derivados por el brazo derecho observados y modelados es de de $2.12 \%$. Se concluye que los valores se consideran aceptables. En lo que respecta a la comparación con los resultados obtenidos por Basile et al. (2015) y Peruzzo et al. (2016), que emplean un modelo bidimensional completo y un modelo cuasi-2D respectivamente, los valores de los errores relativos obtenidos con el modelo que aquí se presenta son similares. Podemos claramente concluir que en la reproducción de la derivación de caudales los tres modelos computan valores con el mismo rango de aproximación.

A su vez, con los resultados obtenidos en las simulaciones para caudales $Q=14310 \mathrm{~m}^{3} / \mathrm{s}$ y $\mathrm{Q}=19570$ $\mathrm{m}^{3} / \mathrm{s}$ se calcularon las distribuciones de caudales específicos en distintas secciones transversales y se compararon con distribuciones observadas en mediciones de campo con ADCP realizadas por la FICH en el período noviembre-diciembre 2004 y febrero-marzo 2006 (FICH, 2006). En las Figuras 3 y 4 se presenta la comparación entre caudales específicos aforados con los resultados obtenidos en las simulaciones para caudales $Q=14310 \mathrm{~m}^{3} / \mathrm{s}$ y $Q=19570$ $\mathrm{m}^{3} / \mathrm{s}$ respectivamente. La calidad del ajuste fue evaluada con el coeficiente de correlación $\mathrm{R}^{2}$, la raíz del error cuadrático medio RECM y el sesgo porcentual PBIAS. En la Tabla 4 se presentan los valores de referencia para evaluar el ajuste según los indicadores utilizados mientras que en las Figuras 3 y 4 se muestran los valores calculados para los mismos y las condiciones alcanzadas en las secciones donde se realizó la comparación. Vale aclarar que valores positivos del PBIAS indican una subestimación por parte del modelo mientras que valores negativos indican sobrestimación. El ajuste según los indicadores resultó entre satisfactorio y muy bueno, considerándolo aceptable. En la Figura 1 se muestra la ubicación de las secciones transversales estudiadas que se corresponden a los $\mathrm{km} 452,440,429.5,429.3,429$ y 427.3.

\begin{tabular}{|c|c|c|c|c|c|c|c|c|}
\hline \multirow[t]{2}{*}{ Fecha } & \multirow[t]{2}{*}{$\begin{array}{c}\mathbf{Q} \\
{\left[\mathbf{m}^{3} / \mathbf{s}\right]}\end{array}$} & \multicolumn{2}{|c|}{$\begin{array}{c}\mathbf{z}_{\mathrm{w}}[\mathrm{m}] \text { IGN } \\
\text { observado }\left(\mathrm{z}_{\mathrm{wo}}\right)\end{array}$} & \multirow[t]{2}{*}{$\underset{\left[\mathbf{s} / \mathbf{m}^{1 / 3}\right]}{\eta^{1 / 3}}$} & \multicolumn{2}{|c|}{$\begin{array}{c}\mathbf{z}_{\mathrm{w}}[\mathrm{m}] \text { IGN } \\
\text { calculado }\left(\mathbf{z}_{\mathrm{wc}}\right)\end{array}$} & \multicolumn{2}{|c|}{$\mathbf{E}_{\mathrm{r}}=100\left|\left(\mathbf{z}_{\mathrm{wc}} / \mathbf{z}_{\mathrm{wo}}\right)-1\right|[\%]$} \\
\hline & & PSM & ROS & & PSM & ROS & PSM & ROS \\
\hline $31 / 08 / 2001$ & 11640 & 5.17 & 4.46 & 0.025 & 5.18 & 4.47 & 0.19 & 0.22 \\
\hline $21 / 02 / 2006$ & 14310 & 6.05 & 5.23 & 0.025 & 6.07 & 5.24 & 0.33 & 0.19 \\
\hline $09 / 06 / 2005$ & 17490 & 7.12 & 6.23 & 0.025 & 7.18 & 6.25 & 0.84 & 0.32 \\
\hline $21 / 12 / 2004$ & 19570 & 7.92 & 6.93 & 0.025 & 7.90 & 6.93 & 0.25 & 0.01 \\
\hline $02 / 04 / 2003$ & 22740 & 8.59 & 7.52 & 0.025 & 8.68 & 7.56 & 1.05 & 0.53 \\
\hline $16 / 08 / 1982$ & 26760 & 9.16 & 8.03 & 0.024 & 9.35 & 8.07 & 2.07 & 0.50 \\
\hline $04 / 01 / 1983$ & 28600 & 9.98 & 8.85 & 0.024 & 10.12 & 8.88 & 1.40 & 0.34 \\
\hline
\end{tabular}

Tabla 2. Porcentaje del caudal total Q derivado por BD y BI en las bifurcaciones para cada simulación - Constitución M04-06.

\begin{tabular}{|c|c|c|c|c|c|c|c|c|c|}
\hline \multirow{2}{*}{ Fecha } & $\begin{array}{c}\text { Q } \\
{\left[\mathbf{m}^{\mathbf{3}} / \mathbf{s}\right]}\end{array}$ & \multicolumn{2}{|c|}{ Bifurcación 1 } & \multicolumn{2}{c|}{ Bifurcación 2 } & \multicolumn{2}{c|}{ Bifurcación 3 } & \multicolumn{2}{c|}{ Bifurcación 4 } \\
\cline { 3 - 10 } & BD (\%) & BI (\%) & BD (\%) & BI (\%) & BD (\%) & BI (\%) & BD (\%) & BI (\%) \\
\hline $31 / 08 / 2001$ & 11640 & 83.13 & 16.87 & 94.33 & 5.67 & 65.88 & 34.12 & 73.42 & 26.58 \\
\hline $21 / 02 / 2006$ & 14310 & 80.93 & 19.07 & 93.10 & 6.90 & 65.07 & 34.93 & 72.80 & 27.20 \\
\hline $09 / 06 / 2005$ & 17490 & 78.86 & 21.14 & 92.25 & 7.75 & 64.02 & 35.98 & 72.63 & 27.37 \\
\hline $21 / 12 / 2004$ & 19570 & 77.62 & 22.38 & 91.75 & 8.25 & 63.39 & 36.61 & 72.88 & 27.12 \\
\hline $02 / 04 / 2003$ & 22740 & 76.39 & 23.61 & 91.39 & 8.61 & 62.73 & 37.27 & 73.15 & 26.85 \\
\hline $16 / 08 / 1982$ & 26760 & 75.44 & 24.56 & 91.01 & 8.99 & 62.25 & 37.75 & 73.44 & 26.56 \\
\hline $04 / 01 / 1983$ & 28600 & 74.33 & 25.67 & 90.51 & 9.49 & 61.63 & 38.37 & 73.87 & 26.13 \\
\hline
\end{tabular}


Tabla 3. Porcentaje del caudal total $Q$ derivado por el brazo derecho en las bifurcaciones del tramo en estudio, contrastación con

\begin{tabular}{|c|c|c|c|c|c|c|c|c|c|c|}
\hline \multirow{2}{*}{$\begin{array}{l}\text { Fecha } \\
\text { Aforo }\end{array}$} & \multirow{2}{*}{$\begin{array}{c}\mathbf{Q} \\
\left(\mathrm{m}^{3} / \mathrm{s}\right)\end{array}$} & \multirow[b]{2}{*}{ Bifurc. } & \multicolumn{2}{|c|}{ \% derivado BD } & \multicolumn{2}{|c|}{ M04-06 } & \multicolumn{2}{|c|}{ Peruzzo et al. (2016) } & \multicolumn{2}{|c|}{ Basile et al. (2015) } \\
\hline & & & Obs. & Calc. & $E_{r}(\%)$ & $\begin{array}{c}\text { Prom. } \\
\left|\mathbf{E}_{\mathbf{r}}\right|(\%)\end{array}$ & $\operatorname{Er}(\%)$ & $\begin{array}{l}\text { Prom. } \\
|\operatorname{Er}|(\%)\end{array}$ & $E_{r}(\%)$ & $\begin{array}{l}\text { Prom. } \\
\left|\mathbf{E}_{\mathbf{r}}\right|(\%)\end{array}$ \\
\hline $30 / 11 / 04$ & 20790 & 1 & 74.30 & 77.53 & 4.35 & \multirow{2}{*}{3.26} & 6.07 & \multirow{2}{*}{4.92} & 5.77 & \multirow{2}{*}{4.36} \\
\hline $21 / 02 / 06$ & 13617 & 1 & 79.40 & 81.12 & 2.17 & & 3.77 & & 2.93 & \\
\hline $30 / 11 / 04$ & 19818 & 2 & 91.20 & 92.15 & 1.04 & \multirow{2}{*}{0.76} & 2.30 & \multirow{2}{*}{2.01} & 1.38 & \multirow{2}{*}{ \pm 1.81} \\
\hline $08 / 03 / 06$ & 14839 & 2 & 92.70 & 93.15 & 0.48 & & 1.73 & & -2.23 & \\
\hline $02 / 12 / 04$ & 19075 & 3 & 61.40 & 64.60 & 5.22 & \multirow{2}{*}{ \pm 3.17} & 2.44 & \multirow{2}{*}{ \pm 3.07} & 4.08 & \multirow{2}{*}{ \pm 2.36} \\
\hline $08 / 03 / 06$ & 14315 & 3 & 66.30 & 65.56 & -1.12 & & -3.70 & & -0.64 & \\
\hline $25 / 11 / 04$ & 16901 & 4 & 70.00 & 71.69 & 2.42 & \multirow{2}{*}{ \pm 1.30} & 2.84 & \multirow{2}{*}{1.57} & 8.80 & \multirow{2}{*}{7.63} \\
\hline $26 / 05 / 06$ & 12634 & 4 & 72.00 & 71.86 & -0.19 & & 0.29 & & 6.47 & \\
\hline
\end{tabular}

Tabla 4. Valores de referencia para la evaluación del ajuste según distintos indicadores.

\begin{tabular}{|c|c|c|c|}
\hline Condición & Coeficiente de Correlación $\mathbf{R}^{2}$ & $\begin{array}{l}\text { Raíz del error cuadrático medio RECM } \\
\qquad\left(\mathrm{m}^{2} / \mathrm{s}\right)\end{array}$ & $\begin{array}{c}\text { Sesgo porcentual } \\
\text { PBIAS }\end{array}$ \\
\hline Muy Bueno (MB) & $\geq 0.85$ & $\leq 1.00$ & \pm 10 \\
\hline Satisfactorio $(\mathrm{S})$ & $0.74-0.50$ & $2.00-3.00$ & $\pm 15- \pm 25$ \\
\hline Pobre $(\mathrm{P})$ & $<0.50$ & $>4.00$ & $> \pm 25$ \\
\hline
\end{tabular}
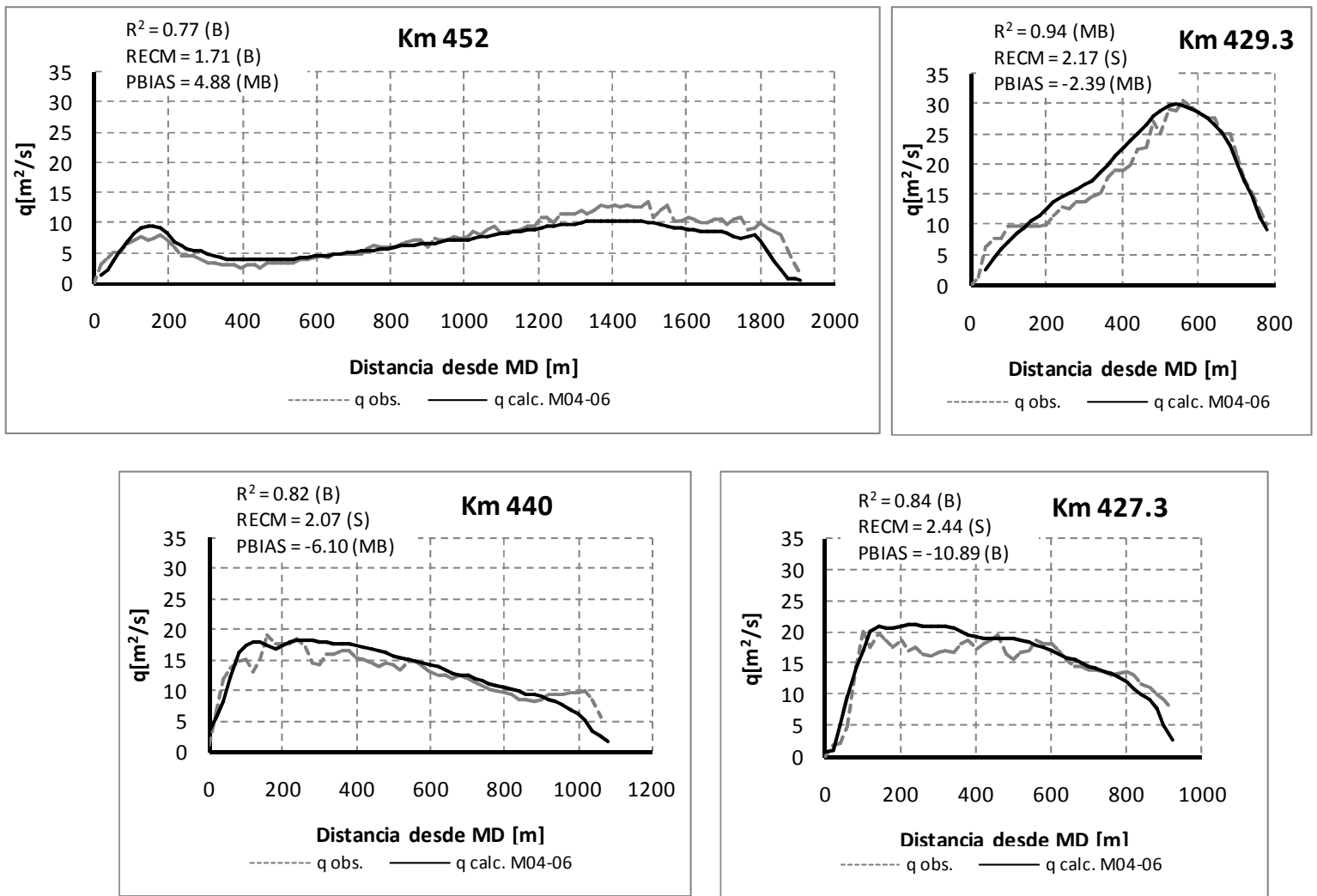

Figura 3. Simulación $Q=14310 \mathrm{~m}^{3} / \mathrm{s}$. Comparación de la distribución de caudales unitarios q calculados y observados (FICH, 2006). 

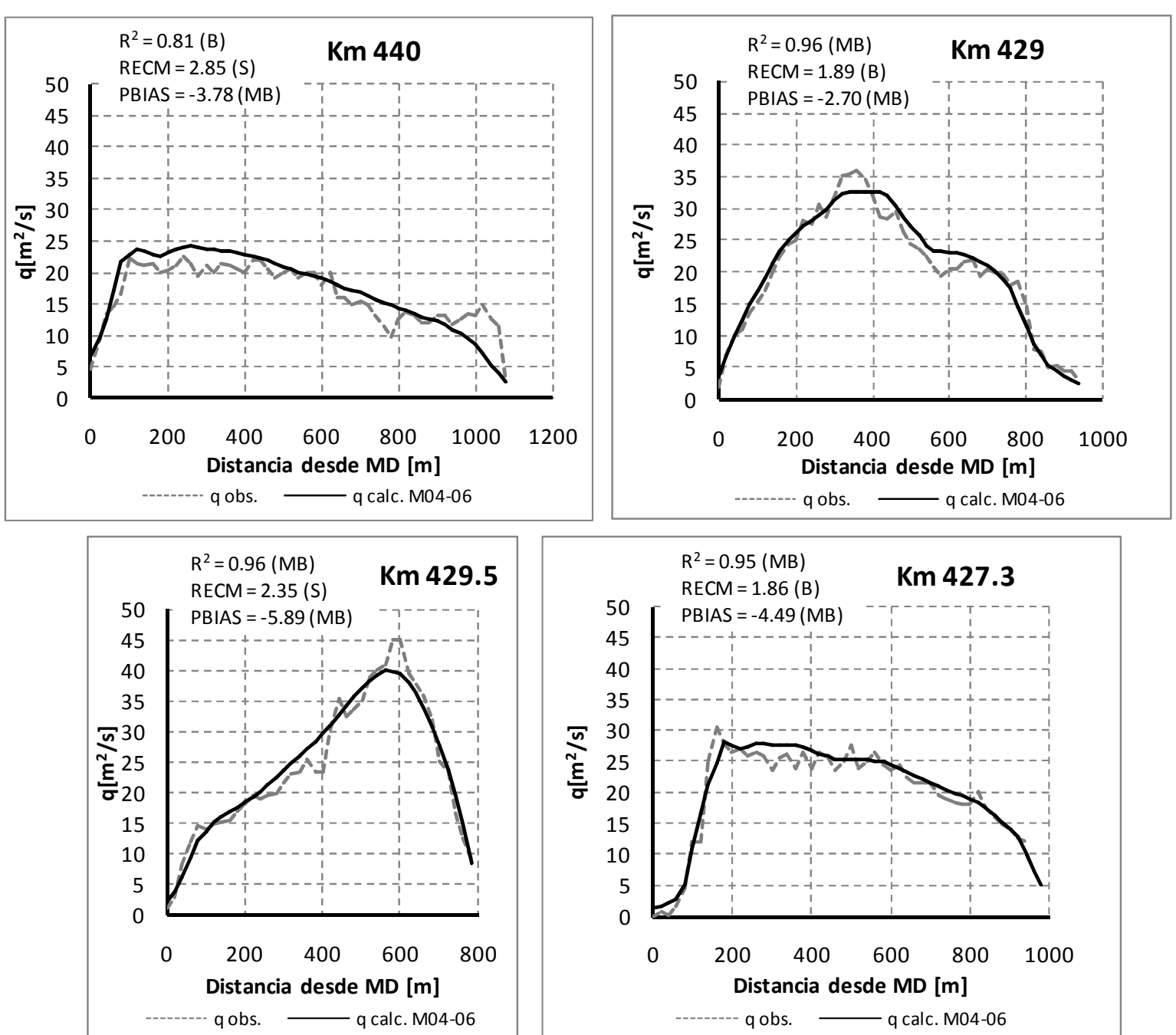

Figura 4. Simulación $Q=19570 \mathrm{~m}^{3} / \mathrm{s}$. Comparación de la distribución de caudales unitarios q calculados y observados (FICH, 2006).

Se realizaron simulaciones en flujo impermanente a modo de validación y recalibración de parámetros de rugosidad reproduciendo la serie de niveles observada en el período 01/01/2004 al 31/12/2004 con un intervalo de integración temporal igual a 10 s. Los caudales simulados estuvieron en el entorno de 13000 a $20000 \mathrm{~m}^{3} / \mathrm{s}$. Se realizaron simulaciones con el coeficiente de rugosidad de Manning variando entre $0.023 \leq \eta \leq 0.025$. Para cuantificar la comparación de la serie de niveles simulada versus la serie observada se utilizaron los siguientes indicadores: el error típico (ET), la eficiencia de Nash-Sutcliffe (NS) y el coeficiente de correlación $\mathrm{R}^{2}$. En la Tabla 4 se presentan los valores de referencia para evaluar el ajuste según los distintos indicadores.

La simulación que mejor calidad de ajuste ha alcanzado al utilizar los calificadores de errores referidos, correspondió a un coeficiente de rugosidad $\eta=0.025$. En la Tabla 5 se muestran los valores calculados de los indicadores para esta simulación y en la Tabla 6 las condiciones alcanzadas según la calificación adoptada. En las Figuras 5 se exponen las variaciones de niveles observadas y simuladas en los dos sitios de comparación para el intervalo de integración temporal. También, en este estado de flujo, los niveles calculados se encuentran dentro del entorno de variación obtenido a partir de caudales y niveles aforados en la estación Paraná-Timbúes.

En régimen impermanente, el modelo reproduce adecuadamente las series de niveles observados en PSM y ROS. El ajuste resultó entre bueno y muy bueno para el intervalo de tiempo de cálculo utilizado en las simulaciones. 
Tabla 5. Valores de referencia para la evaluación del ajuste según distintos indicadores.

\begin{tabular}{|l|c|c|c|}
\hline \multicolumn{1}{|c|}{ Condición } & Error Típico ET (m) & Eficiencia de Nash-Sutcliffe NS & ${\text { Coeficiente de Correlación } \mathbf{R}^{\mathbf{2}}}$ \\
\hline Muy Bueno (MB) & $\leq 0.05$ & $\geq 0.75$ & 0.85 \\
\hline Bueno (B) & $0.05-0.10$ & $0.74-0.65$ & $0.84-0.65$ \\
\hline Satisfactorio (S) & $0.10-0.15$ & $0.64-0.50$ & $0.74-0.50$ \\
\hline Pobre (P) & $>0.15$ & $<0.50$ & $<0.50$ \\
\hline
\end{tabular}

Tabla 6. Valores de indicadores de error en simulación con $\eta$ $=0.025$ - Constitución M04-06.

\begin{tabular}{|c|c|c|}
\hline \multirow{2}{*}{ Indicador } & \multicolumn{2}{|c|}{$\Delta \mathbf{t}=\mathbf{1 0} \mathbf{~}$} \\
\cline { 2 - 3 } & $\mathbf{P S M}$ & $\mathbf{R O S}$ \\
\hline Error Típico & 0.084 & 0.093 \\
\hline $\mathrm{NS}$ & 0.955 & 0.973 \\
\hline $\mathrm{R}^{2}$ & 0.988 & 0.981 \\
\hline
\end{tabular}

Tabla 7. Calificación de los errores para simulación con $\eta=$

\begin{tabular}{|c|c|c|}
\multicolumn{2}{c}{0.025} & - Constitución M04-06. \\
\hline \multirow{2}{*}{ Indicador } & \multicolumn{2}{|c|}{$\Delta \mathbf{t}=\mathbf{1 0}$ s } \\
\cline { 2 - 3 } & PSM & ROS \\
\hline Error Típico & $\mathrm{B}$ & $\mathrm{B}$ \\
\hline $\mathrm{NS}$ & $\mathrm{MB}$ & $\mathrm{MB}$ \\
\hline $\mathrm{R}^{2}$ & $\mathrm{MB}$ & $\mathrm{MB}$ \\
\hline
\end{tabular}

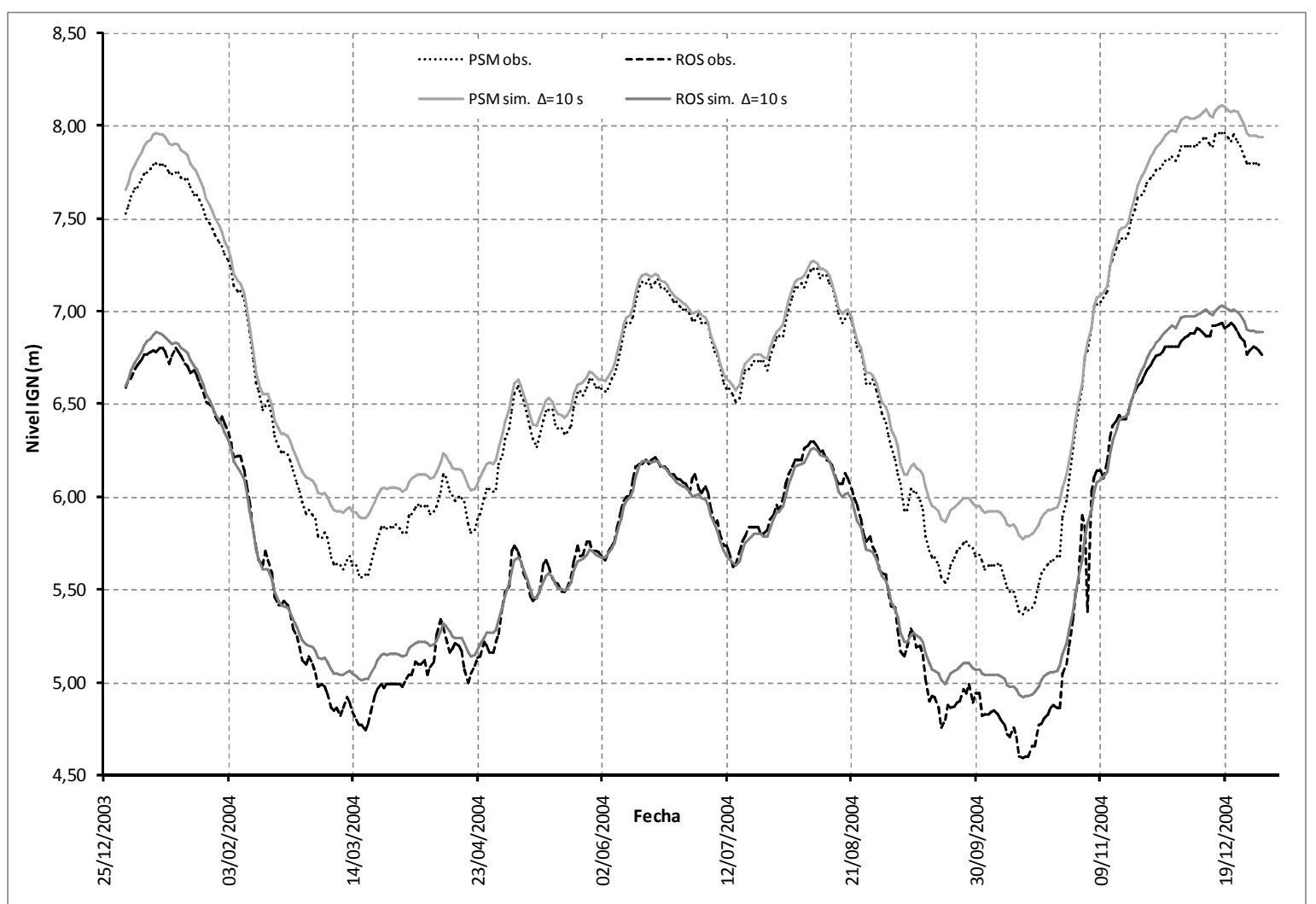

Figura 5. Perfiles de superficie libre observados y simulados para la serie 01/01/2004 al 31/12/2004 - Constitución M04-06.

\section{Resultados de las simulaciones de la Constitución M10-12}

En flujo permanente, el rango de valores del coeficiente de rugosidad de Manning que proporcionó el mejor ajuste en términos de niveles fue de 0.023 . El error relativo porcentual entre niveles observados y simulados se acota en $1.22 \%$, siendo el valor medio $0.41 \%$. Los niveles calculados se encuentran dentro del entorno de variación obtenido a partir de caudales y niveles aforados por la Subsecretaría de Recursos Hídricos de la Nación en la estación Paraná-Timbúes en el período 2000-2016. En la Tabla 8 se muestra una síntesis de los parámetros observados y calculados para cada simulación.

Para estudiar la distribución de caudales en las bifurcaciones del dominio se contaba con caudales 
aforados en las tres primeras durante la campaña realizada por UNIBO - FICH los días 29 de junio a 3 de julio de 2009. En primer lugar, se estimaron los caudales derivados en las distintas secciones para cada uno de los 7 escenarios de flujo permanente simulados. En la Tabla 9 se presentan los porcentajes del caudal total Q derivados en cada bifurcación para el brazo derecho (BD) y para el brazo izquierdo (BI), para cada simulación. Al igual que en la constitución M04-06, se observa que en las secciones correspondientes a los $\mathrm{km}$ 449, 440 y 436 el porcentaje del caudal total derivado por el brazo derecho es superior para aguas bajas que para aguas altas, mientras que en la sección correspondiente al $\mathrm{km} 416.5$ este porcentaje es similar para ambos estados. Los caudales aforados por UNIBO - FICH (Kazimierski, 2014) en las bifurcaciones corresponden a caudales distintos a los empleados en las simulaciones para la etapa de calibración. Para poder determinar los valores a comparar con los aforos, se procedió a ajustar relaciones lineales entre caudal total Q simulado versus los respectivos valores de porcentaje de caudal derivado por cada brazo en cada bifurcación. Una vez halladas estas relaciones se obtuvieron los porcentajes en función de los caudales aforados para luego calcular los errores relativos de su comparación con los porcentajes observados. En la Tabla 10 se presentan los resultados de las comparaciones realizadas en el brazo derecho para las tres primeras bifurcaciones. El error relativo porcentual entre porcentaje de caudal observado y simulado se acota en $5.83 \%$, siendo el valor medio $2.51 \%$. Se concluye que los valores se consideran aceptables.
A su vez, se contaba con perfiles transversales de velocidad medidos con ADCP en distintas secciones del dominio durante la misma campaña mencionada anteriormente (Kazimierski, 2014). Se procedió a realizar una simulación representativa de los días de campaña para poder comparar los perfiles observados. Como condición de borde aguas arriba se impuso un caudal de ingreso igual a $13740 \mathrm{~m}^{3} / \mathrm{s}$ y aguas abajo una altura de agua igual a $4.48 \mathrm{~m}$. Con los resultados obtenidos en la simulación se calcularon los perfiles de velocidad en las distintas secciones transversales donde se contaba con valores observados. En la Figura 6 se presenta la comparación entre perfiles de velocidad aforados en el brazo derecho (BD) y en el brazo izquierdo (BI) con los resultados obtenidos en las simulaciones para caudal $Q=13740 \mathrm{~m}^{3} / \mathrm{s}$. La calidad del ajuste fue evaluada con el coeficiente de correlación $\mathrm{R}^{2}$, la raíz del error cuadrático medio RECM y el sesgo porcentual PBIAS. En la Tabla 11 se presentan los valores de referencia para evaluar el ajuste según los indicadores utilizados mientras que en las tablas 12 y 13 se muestran los valores calculados para los mismos y las condiciones alcanzadas en las secciones donde se realizó la comparación. Vale aclarar que valores positivos del PBIAS indican una subestimación por parte del modelo mientras que valores negativos indican sobrestimación. El ajuste según los indicadores resultó entre satisfactorio y muy bueno, considerándolo aceptable. En la Figura 1 se muestra la ubicación de las secciones transversales estudiadas que se corresponden a los $\mathrm{km} 447,442$, y 434.

Tabla 8. Niveles Observados, Calculados y Errores porcentuales para constitución M10-12.

\begin{tabular}{|c|c|c|c|c|c|c|c|c|}
\hline \multirow[t]{2}{*}{ Fecha } & \multirow[t]{2}{*}{$\begin{array}{c}\mathbf{Q} \\
{\left[\mathrm{m}^{3} / \mathrm{s}\right]}\end{array}$} & \multicolumn{2}{|c|}{$\begin{array}{c}\mathbf{z}_{\mathrm{w}}[\mathrm{m}] \text { IGN } \\
\text { observado }\left(\mathrm{z}_{\mathrm{wo}}\right)\end{array}$} & \multirow[t]{2}{*}{$\underset{\left[\mathbf{s} / \mathbf{m}^{1 / 3}\right]}{\eta^{1 / 3}}$} & \multicolumn{2}{|c|}{$\begin{array}{c}\mathrm{z}_{\mathrm{w}}[\mathrm{m}] \text { IGN } \\
\text { calculado }\left(\mathrm{z}_{\mathrm{wc}}\right)\end{array}$} & \multicolumn{2}{|c|}{$\mathbf{E}_{\mathrm{r}}=100\left|\left(\mathrm{z}_{\mathrm{wc}} / \mathbf{z}_{\mathrm{wo}}\right)-1\right|[\%]$} \\
\hline & & PSM & ROS & & PSM & ROS & PSM & ROS \\
\hline $04 / 10 / 2010$ & 14460 & 5.90 & 5.06 & 0.023 & 5.93 & 5.05 & 0.43 & 0.17 \\
\hline $12 / 12 / 2013$ & 16688 & 6.80 & 5.99 & 0.023 & 6.88 & 5.99 & 1.22 & 0.02 \\
\hline $29 / 06 / 2012$ & 17309 & 7.07 & 6.16 & 0.023 & 7.06 & 6.14 & 0.13 & 0.40 \\
\hline $16 / 10 / 2014$ & 19096 & 7.77 & 6.88 & 0.023 & 7.78 & 6.85 & 0.13 & 0.44 \\
\hline $18 / 12 / 2015$ & 21602 & 8.43 & 7.35 & 0.024 & 8.44 & 7.32 & 0.07 & 0.41 \\
\hline $19 / 08 / 2014$ & 22199 & 8.66 & 7.74 & 0.023 & 8.74 & 7.72 & 0.92 & 0.26 \\
\hline $18 / 02 / 2010$ & 24628 & 9.52 & 8.58 & 0.023 & 9.61 & 8.56 & 0.95 & 0.23 \\
\hline
\end{tabular}

Tabla 9. Porcentaje del caudal total Q derivado por BD y BI en las bifurcaciones para cada simulación - Constitución M10-12.

\begin{tabular}{|c|c|c|c|c|c|c|c|c|c|}
\hline \multirow{2}{*}{ Fecha } & $\mathbf{Q}$ & \multicolumn{2}{|c|}{ Bifurcación 1 } & \multicolumn{2}{c|}{ Bifurcación 2 } & \multicolumn{2}{c|}{ Bifurcación 3 } & \multicolumn{2}{c|}{ Bifurcación 4 } \\
\cline { 3 - 10 } & {$\left[\mathbf{m}^{\mathbf{3}} / \mathbf{s}\right]$} & BD (\%) & BI (\%) & BD (\%) & BI (\%) & BD (\%) & BI (\%) & BD (\%) & BI (\%) \\
\hline $04 / 10 / 2010$ & 14460 & 83.16 & 16.84 & 96.62 & 3.38 & 69.78 & 30.22 & 75.78 & 22.30 \\
\hline $12 / 12 / 2013$ & 16688 & 80.43 & 19.57 & 94.91 & 5.09 & 68.18 & 31.82 & 75.53 & 22.36 \\
\hline $29 / 06 / 2012$ & 17309 & 79.99 & 20.01 & 94.62 & 5.38 & 67.97 & 32.03 & 75.65 & 22.30 \\
\hline $16 / 10 / 2014$ & 19096 & 78.33 & 21.67 & 93.85 & 6.15 & 67.28 & 32.72 & 75.41 & 22.53 \\
\hline $18 / 12 / 2015$ & 21602 & 76.99 & 23.01 & 93.27 & 6.73 & 66.80 & 33.20 & 75.27 & 22.64 \\
\hline $19 / 08 / 2014$ & 22199 & 76.31 & 23.69 & 93.03 & 6.97 & 66.54 & 33.46 & 75.37 & 22.52 \\
\hline $18 / 02 / 2010$ & 24628 & 74.78 & 25.22 & 92.40 & 7.60 & 66.00 & 34.00 & 75.33 & 22.61 \\
\hline
\end{tabular}


Tabla 10. Porcentaje del caudal total $Q$ derivado por el brazo derecho en las bifurcaciones del tramo en estudio, contrastación con datos observados.

\begin{tabular}{|c|c|c|c|c|c|}
\hline \multirow{2}{*}{ Fecha Aforo } & \multirow{2}{*}{$\left.\mathbf{( m}^{\mathbf{3}} / \mathbf{s}\right)$} & \multirow{2}{*}{ Bifurc. } & \multicolumn{2}{|c|}{ \% derivado BD } & \multirow{2}{*}{$\mathbf{E}_{\mathbf{r}}(\mathbf{\%})$} \\
\hline \multirow{2}{*}{29 de junio - 03 de julio, 2009 } & & & Obs. & Calc. & \\
\cline { 4 - 6 } & 13224 & 1 & 79.28 & 83.90 & 5.83 \\
\cline { 2 - 6 } & 13910 & 2 & 94.87 & 96.29 & 1.50 \\
\cline { 2 - 6 } & 13408 & 3 & 69.38 & 69.52 & 0.21 \\
\hline
\end{tabular}
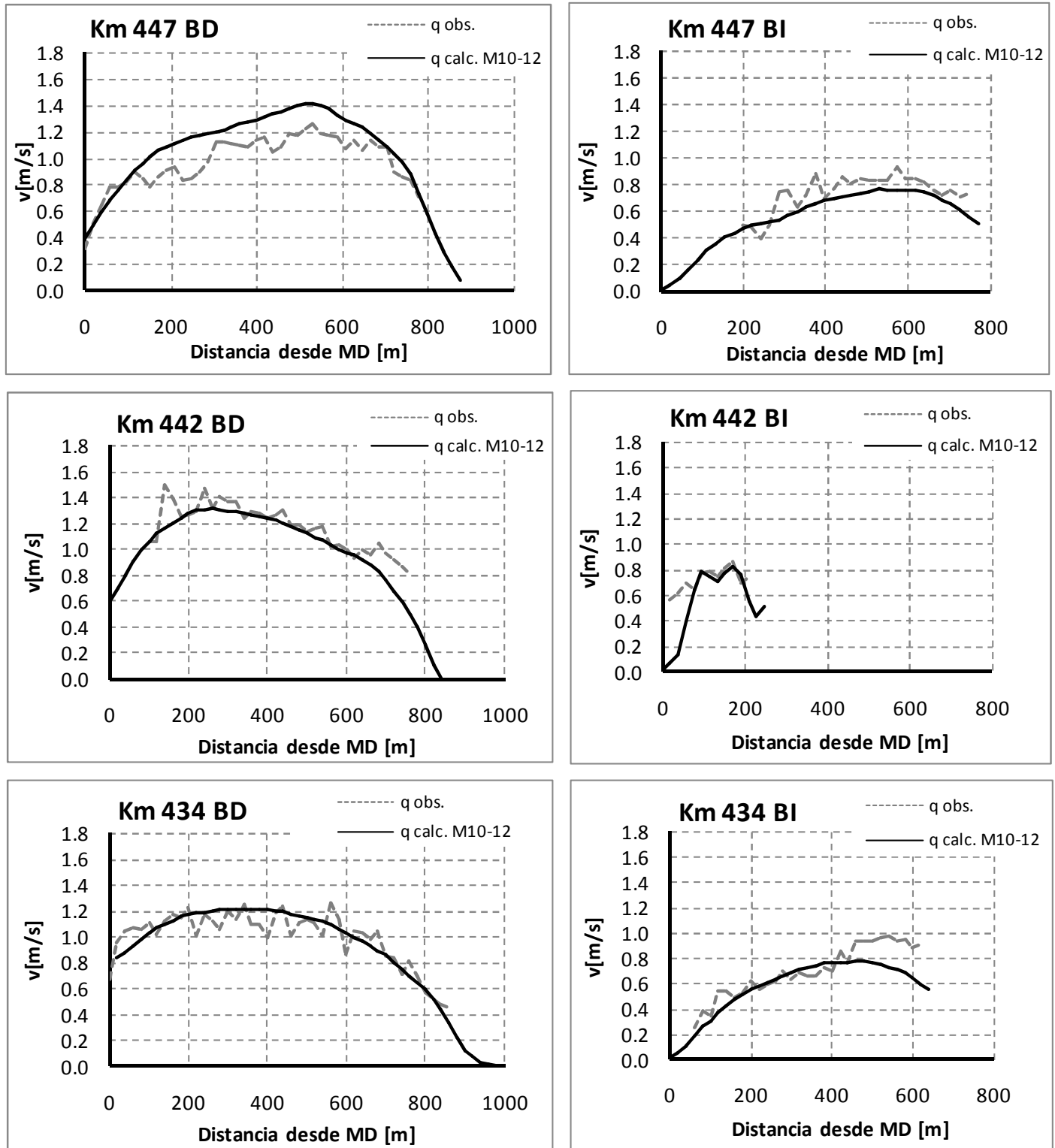

Figura 6. $Q=13740 \mathrm{~m}^{3} / \mathrm{s}$. Comparación entre perfiles de velocidad simulados y observados (Kazimierski, 2014).

Tabla 11. Valores de referencia para la evaluación del ajuste según distintos indicadores.

\begin{tabular}{|l|c|c|c|}
\hline \multicolumn{1}{|c|}{ Condición } & Coeficiente de Correlación $\mathbf{R}^{\mathbf{2}}$ & $\begin{array}{c}\text { Raíz del error cuadrático medio RECM } \\
(\mathbf{m} / \mathbf{s})\end{array}$ & $\begin{array}{c}\text { Sesgo porcentual } \\
\text { PBIAS }\end{array}$ \\
\hline Muy Bueno (MB) & $\geq 0.85$ & $\leq 0.10$ & \pm 10 \\
\hline Bueno (B) & $0.84-0.65$ & $0.10-0.20$ & $\pm 10- \pm 15$ \\
\hline Satisfactorio (S) & $0.74-0.50$ & $0.20-0.30$ & $\pm 15- \pm 25$ \\
\hline Pobre (P) & $<0.50$ & $>0.30$ & $> \pm 25$ \\
\hline
\end{tabular}


Tabla 12. Valores de indicadores de error - Comparación entre perfiles de velocidad simulados y observados.

\begin{tabular}{|c|c|c|c|c|c|c|}
\hline \multirow{2}{*}{ Indicador } & \multicolumn{6}{|c|}{ Sección transversal } \\
\hline & km 447 BD & km 447 BI & km 442 BD & km 442 BI & km 434 BD & km 434 BI \\
\hline $\mathrm{R}^{2}$ & 0.87 & 0.71 & 0.81 & 0.67 & 0.82 & 0.75 \\
\hline RECM & 0.17 & 0.11 & 0.13 & 0.24 & 0.10 & 0.14 \\
\hline PBIAS & -13.77 & 10.11 & 7.32 & 21.74 & 0.19 & 14.87 \\
\hline
\end{tabular}

Tabla 13. Calificación de los errores - Comparación entre perfiles de velocidad simulados y observados.

\begin{tabular}{|c|c|c|c|c|c|c|}
\hline \multirow{2}{*}{ Indicador } & \multicolumn{6}{|c|}{ Sección transversal } \\
\cline { 2 - 7 } & $\mathbf{k m}$ 447 BD & $\mathbf{k m}$ 447 BI & $\mathbf{k m ~ 4 4 2} \mathbf{~ B D}$ & $\mathbf{k m ~ 4 4 2}$ BI & $\mathbf{k m ~ 4 3 4 ~ B D ~}$ & $\mathbf{k m ~ 4 3 4}$ BI \\
\hline $\mathrm{R}^{2}$ & $\mathrm{MB}$ & $\mathrm{B}$ & $\mathrm{B}$ & $\mathrm{B}$ & $\mathrm{B}$ & $\mathrm{B}$ \\
\hline RECM & $\mathrm{B}$ & $\mathrm{B}$ & $\mathrm{B}$ & $\mathrm{S}$ & $\mathrm{MB}$ & $\mathrm{B}$ \\
\hline PBIAS & $\mathrm{B}$ & $\mathrm{B}$ & $\mathrm{MB}$ & $\mathrm{S}$ & $\mathrm{MB}$ & $\mathrm{B}$ \\
\hline
\end{tabular}

Se realizaron simulaciones en flujo impermanente a modo de validación y recalibración de parámetros de rugosidad reproduciendo la serie de niveles observada en el período 16/03/2015 al 15/03/2016 con un intervalo de integración temporal igual a $10 \mathrm{~s}$. Los caudales simulados estuvieron en el entorno de 16000 a 26000 $\mathrm{m}^{3} / \mathrm{s}$. Se realizaron simulaciones con el coeficiente de rugosidad de Manning variando entre $0.023 \leq \eta \leq 0.025$. Para cuantificar la comparación de la serie de niveles simulada versus la serie observada se utilizaron los mismos indicadores que en la constitución M04-06 (Tabla 5). La simulación que mejor calidad de ajuste ha alcanzado al utilizar los calificadores de errores referidos, correspondió a un coeficiente de rugosidad $\eta=$ 0.023 . En la Tabla 14 se muestran los valores calculados de los indicadores para esta simulación y en la Tabla 15 las condiciones alcanzadas según la calificación adoptada. En la Figura 7 se exponen las variaciones de niveles observadas y simuladas en los dos sitios de comparación para el intervalo de integración temporal. También, en este estado de flujo, los niveles calculados se encuentran dentro del entorno de variación obtenido a partir de caudales y niveles aforados en la estación Paraná-Timbúes.

En régimen impermanente, el modelo reproduce adecuadamente las series de niveles observados en PSM y ROS. El ajuste resultó entre bueno y muy bueno para el intervalo de tiempo de cálculo utilizado en las simulaciones.

Evolución temporal de la distribución de caudales en las bifurcaciones

A partir de los resultados presentados en las Tablas 2 y 9 se realizó un análisis conjunto que se muestra en la Figura 7. En la misma se muestran en forma conjunta los porcentajes del caudal total Q derivados en cada bifurcación para el brazo derecho (BD) y para el brazo izquierdo (BI) para los escenarios de flujo permanente simulados en ambas constituciones. Como puede observase, los caudales Q empleados en las simulaciones en flujo permanente fueron distintos para una constitución que para la otra. Para realizar el análisis de la evolución temporal de la partición de caudales en las bifurcaciones se evaluaron las relaciones lineales, mencionadas en los apartados anteriores, que permiten obtener el porcentaje de caudal total derivado por el brazo derecho en cada bifurcación para dos estados de aguas. Un estado de aguas medias bajas y otro de aguas medias altas, con caudales totales de $14000 \mathrm{~m}^{3} / \mathrm{s}$ y 20000 $\mathrm{m}^{3} / \mathrm{s}$ respectivamente. Mediante dichas relaciones se calcularon los porcentajes para ambos estados en cada constitución y se determinaron las diferencias entre los mismos. En la Tabla 16 se presentan los resultados de las comparaciones realizadas.

Tabla 14. Valores de indicadores de error en simulación con $\eta=$ 0.023 - Constitución M10-12.

\begin{tabular}{|c|c|c|}
\hline \multirow{2}{*}{ Indicador } & \multicolumn{2}{|c|}{$\Delta \mathbf{t}=\mathbf{1 0} \mathbf{~ s}$} \\
\cline { 2 - 3 } & $\mathbf{P S M}$ & $\mathbf{R O S}$ \\
\hline Error Típico & 0.049 & 0.056 \\
\hline $\mathrm{NS}$ & 0.990 & 0.996 \\
\hline $\mathrm{R}^{2}$ & 0.998 & 0.996 \\
\hline
\end{tabular}

Tabla 15. Calificación de los errores para simulación con $\eta=$ 0.023 - Constitución M10-12.

\begin{tabular}{|c|c|c|}
\hline \multirow{2}{*}{ Indicador } & \multicolumn{2}{|c|}{$\Delta \mathbf{t}=\mathbf{1 0} \mathbf{~}$} \\
\cline { 2 - 3 } & $\mathbf{P S M}$ & $\mathbf{R O S}$ \\
\hline Error Típico & $\mathrm{MB}$ & $\mathrm{B}$ \\
\hline $\mathrm{NS}$ & $\mathrm{MB}$ & $\mathrm{MB}$ \\
\hline $\mathrm{R}^{2}$ & $\mathrm{MB}$ & $\mathrm{MB}$ \\
\hline
\end{tabular}

En cuanto a la partición de caudales en las cuatro bifurcaciones que se presentan en el tramo se observa un incremento de los porcentajes del caudal total derivado por el brazo derecho, y una correspondiente disminución por el brazo izquierdo. El caudal derivado por el brazo derecho se incrementa en un rango de un $2.35 \%$ a $3.92 \%$ en aguas bajas a un rango entre $0.55 \%$ a $3.87 \%$ en aguas altas. 


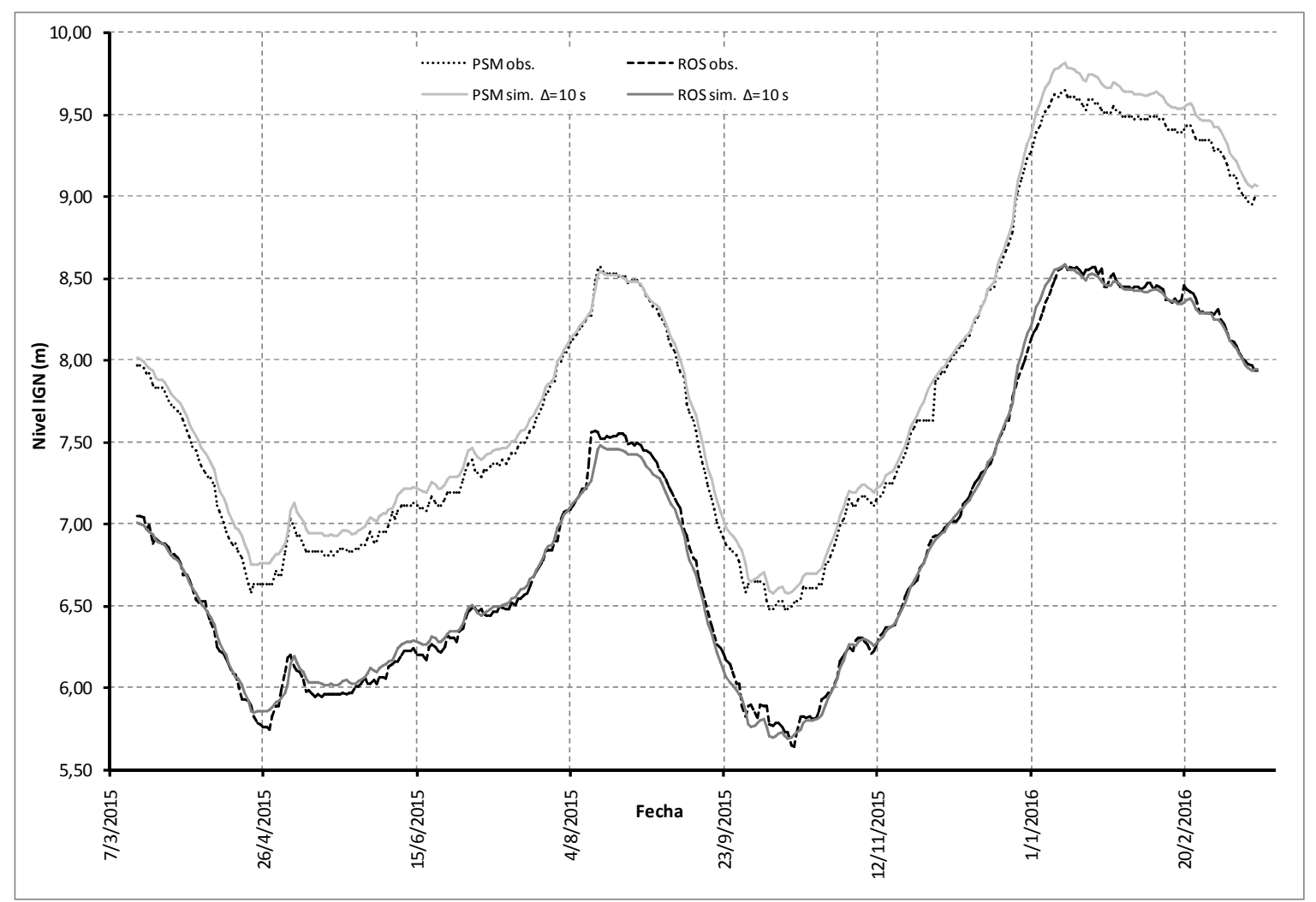

Figura 7. Perfiles de superficie libre observados y simulados para serie 16/03/2015 al 15/03/2016 - Constitución M10-12.
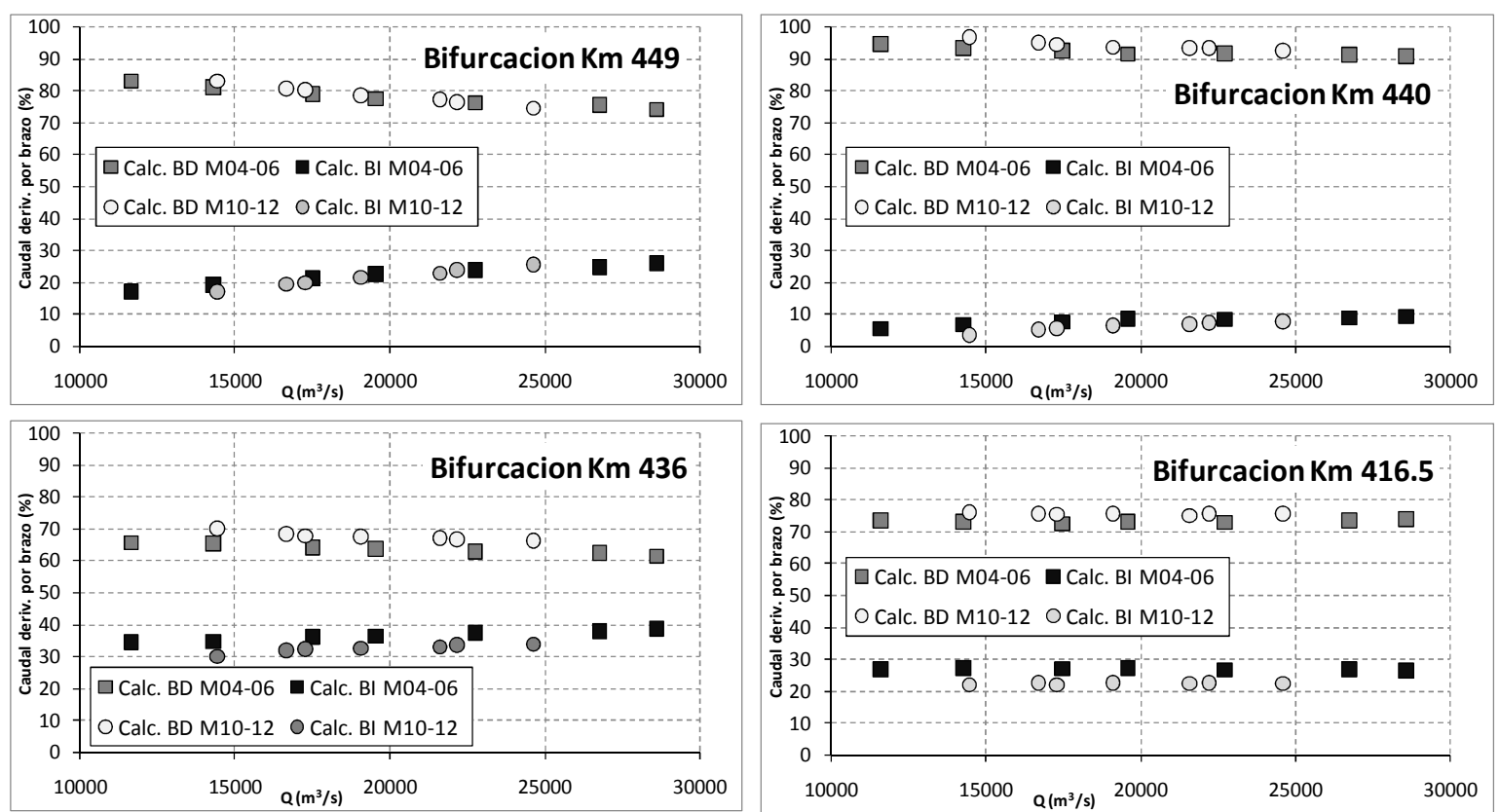

Figura 8. Comparación de los porcentajes del caudal total Q derivado por el BD y el BI calculados para cada uno de los 7 escenarios de flujo permanente simulados para las constituciones M04-06 y M10-12. 
Tabla 16. Comparación entre porcentajes de caudal total $Q$ derivado por el brazo derecho calculados para dos estados de agua

\begin{tabular}{|c|c|c|c|c|}
\hline \multirow{2}{*}{$\begin{array}{c}Q \\
\left(\mathrm{~m}^{3} / \mathrm{s}\right)\end{array}$} & \multirow[b]{2}{*}{ Bifurcación } & \multicolumn{2}{|c|}{ \% derivado BD } & \multirow[b]{2}{*}{ Dif. \% deriv. BD } \\
\hline & & M04-06 & M10-12 & \\
\hline 14000 & $\mathrm{~km} 449$ & 80.93 & 83.28 & 2.35 \\
\hline 20000 & $\mathrm{~km} 449$ & 77.93 & 78.48 & 0.55 \\
\hline 14000 & $\mathrm{~km} 440$ & 93.31 & 96.25 & 2.94 \\
\hline 20000 & $\mathrm{~km} 440$ & 92.11 & 93.95 & 1.84 \\
\hline 14000 & $\mathrm{~km} 436$ & 65.62 & 69.32 & 3.70 \\
\hline 20000 & $\mathrm{~km} 436$ & 64.42 & 67.32 & 2.90 \\
\hline 14000 & $\mathrm{~km} 416.6$ & 71.81 & 75.73 & 3.92 \\
\hline 20000 & $\mathrm{~km} 416.6$ & 71.57 & 75.44 & 3.87 \\
\hline
\end{tabular}

\section{CONCLUSIONES}

El modelo bidimensional TELEMAC-2D fue implementado para simular la hidrodinámica en régimen permanente e impermanente de un tramo del río Paraná Inferior para dos configuraciones morfológicas correspondientes a los periodos de años 2004-2006 y 2010-2012. El rango de caudales de caudales que involucró el estudio para la constitución 2004-2006 se valorizó entre 11600 a $28600 \mathrm{~m}^{3} / \mathrm{s}$, en tanto que para el período $2010-2012$ los caudales simulados estuvieron en el entorno de 14400 a $24600 \mathrm{~m}^{3} / \mathrm{s}$.

A partir del grado de aproximación obtenido entre niveles y caudales observados versus simulados se concluye que los resultados han sido aceptables, lográndose reproducir en forma satisfactoria niveles hidrométricos en 2 puntos de contrastación y derivación de caudales en 4 bifurcaciones del tramo. Particularmente en la constitución M04-06 el modelo demostró una buena capacidad en reproducir caudales específicos en 6 secciones transversales del dominio, mientras que en la constitución M10-12 se reprodujeron satisfactoriamente perfiles de velocidad también en 6 secciones transversales.

El análisis específico de la partición de caudales en las bifurcaciones del tramo evidencia un incremento de los porcentajes del caudal derivado por el brazo derecho, y una correspondiente disminución por el brazo izquierdo en las cuatro bifurcaciones analizadas, en el período de 2004 a 2010.

Se concluye que el modelo bidimensional aplicado es capaz de describir satisfactoriamente la hidrodinámica del tramo en estudio con una aceptable aproximación de variables de flujo tales como niveles, caudales, velocidades y caudales específicos, haciéndolo particularmente apto como base hidrodinámica para la descripción de diversos procesos presentes en la morfodinámica de este sistema fluvial.

\section{AGRADECIMIENTOS}

Los autores desean expresar su agradecimiento a la UNR por el apoyo brindado en el marco de los PID 1ING509 Y 1ING514 y a la DNVN-DPI por los datos topobatimétricos e hidrológicos facilitados.

\section{BIBLIOGRAFÍA}

Basile, P.A., Riccardi, G.A., Peruzzo, F. y Trivisonno F. 2015. Modelación Hidrodinámica de un tramo del Río Paraná Inferior. Séptimo Simposio Regional sobre Hidráulica de Ríos. Montevideo, Uruguay. E-Book

BCR. 2015. Informativo seminal BCR. Bolsa de Comercio de Rosario. Año XXXIII - N 1707 - 15 de Mayo de 2015. Rosario, Argentina. 36 p.

CHC-NRC. 2011. Blue Kenue, Reference Manual. Canadian Hydraulics Centre - National Research Council, Canada.

DNVN-DPI. 2014. Cartografía del río Paraná. Datos topobatimétricos tramo $\mathrm{km} 232$ - 480. Dirección Nacional de Vías Navegables Delegación Paraná Inferior, Rosario.

FICH. 2004. Estudios Hidráulicos y Morfológicos Zona Isla de la Invernada, Río Paraná. Informe final. Facultad de Ingeniería y Ciencias Hídricas, UNL.

FICH. 2006. Estudios Complementarios Hidráulicos y Morfológicos de la Zona de la Isla de la Invernada. Informe final. Facultad de Ingeniería y Ciencias Hídricas, UNL.

Galland, J.-C., Goutal, N., Hervouet, J.-M. 1991. TELEMAC: A new numerical model for solving shallow water equations. Advances in Water 
Resources, Vol. 14, № 3, pp. 138-148.

Garcia, M., Basile, P.A., Riccardi, G. y Rodríguez, J.F. 2013. Modelling Hydrodynamic and Sedimentation Processes in Large Lowland Rivers: An Application to the Paraná River (Argentina). 35th IAHR World Congress, Chengdu, China.

Guerrero, M. y Lamberti, A. 2013. Bed-roughness investigation for a 2-D model calibration: the San Martín case study at Lower Paraná. International Journal of Sediment Research, Vol. 28, No 4, pp. 458-469.

Hervouet, J.M. 2000. TELEMAC modelling system: an overview. Hydrological Processes, Vol. 14, $\mathrm{N}^{\mathrm{a}}$ 13, pp. 2209-2210.

Lang, P., Desombre, J., Ata, R., Goeury, C., Hervouet, J.M. 2014. TELEMAC-2D Software, User Manual.

Peruzzo, F., Riccardi, G., Basile, P.A. 2016. Modelación cuasi-bidimensional de escurrimiento superficial en cauce principal del tramo Paraná inferior km 410-452. Encuentro de Investigadores en Formación en Recursos Hídricos IFRH2016, Ezeiza, Argentina.

SAyDS. 2013. Inventario de los humedales de Argentina: Sistemas de paisajes de humedales del Corredor Fluvial Paraná - Paraguay. Secretaria de Ambiente y Desarrollo Sustentable, Proyecto GEF 4206 - PNUD ARG/10/003, $1^{\circ}$ Edición, Buenos Aires, Argentina.
SHN 2014. Base de Datos Digital de Cartas Náuticas de Río Paraná Inferior. Servicio de Hidrografía Naval, http://www.hidro.gov.ar/cartas/cartasnauticas.asp.

Singh, V.P. y Woolhiser, D.A. 2002. Mathematical Modeling of Watershed Hydrology. Journal of Hydrologic Engineering, Vol. 7, No 4, pp. 270-292.

Taller Ecologista 2010. Humedales del Paraná. Biodiversidad, usos y amenazas en el Delta Medio. Proyecto: Iniciativas sustentables en los humedales del Paraná Inferior, financiado por el Programa de Subvenciones para Ecosistemas (EGP) del Comité Holandés para la IUCN NL (Comité Nacional de los Países Bajos). Edición Inercia Comunicaciones. Rosario, Argentina. 68 p. Kazimierski, L. D. 2014. Sedimentación en canales de navegación. caso de estudio: paso borghi. Tesis de grado, FI, UBA, Buenos Aires, Argentina. 143 p.

Artículo recibido el 03/2018 y aprobado para su publicación el 06/2018. 